\title{
Self-administered questionnaire versus interview as a screening method for intimate partner violence in the prenatal setting in Japan: A randomised controlled trial
}

\author{
Yaeko Kataoka ${ }^{1 *}$, Yukari Yaju², Hiromi Eto ${ }^{1}$, Shigeko Horiuchi ${ }^{1}$
}

\begin{abstract}
Background: Intimate partner violence (IPV) is a serious social issue in Japan. In order to start effective interventions for abused women, the appropriate method of screening for IPV in healthcare settings needs clarifying. The objective of this study was to compare the effectiveness of a face-to-face interview with a selfadministered questionnaire. We used the Violence Against Women Screen (VAWS), a Japanese screening instrument for intimate partner violence (IPV), for identifying pregnant women who have experienced abuse.

Methods: We conducted a randomised controlled trial to screen participants at three points in time in a prenatal clinic in Tokyo, Japan. There were 328 consenting women between 14 and 25 weeks of pregnancy who were consecutively selected and randomly assigned to either the interview or self-administered questionnaire group. Both groups completed the same screening instrument three times during their pregnancy. The primary outcome was the total number of women identified by each screening method and the secondary outcome was the effect of the screening as measured by the women's comfort level and their expressed need to consult with the nurse.

Results: For all three screenings, the identification rate in the interview group was significantly lower than that for the self-administered questionnaire group (relative risk $0.66,95 \% \mathrm{Cl} 0.46$ to 0.97 ), even after controlling for smoking (adjusted odds ratio $0.59,95 \% \mathrm{Cl} 0.35$ to 0.98 ). The two groups did not differ for secondary outcomes.
\end{abstract}

Conclusions: The self-administered questionnaire identified more IPV than the face-to-face interview when screening pregnant women in a Japanese prenatal clinic.

Trial Registration: UMIN-CTRC0000000353.

\section{Background}

Intimate partner violence (IPV) is a serious social issue in Japan. A national survey conducted in 2008 revealed that approximately $25 \%$ of adult women, an estimated eight million women, reported being subjected to physical violence by a male partner, over their lifetime: approximately $16 \%$ reported sexual violence, and approximately $17 \%$ reported psychological abuse [1]. This survey revealed that the 5-year prevalence of IPV was $14 \%$ among women. Furthermore the number of

\footnotetext{
* Correspondence: yaeko-kataoka@slcn.ac.jp

'St Luke's College of Nursing, 10-1 Akashi-cho, Chuo-ku, Tokyo 104-0044,

Japan Associate Professor in nursing and midwifery

Full list of author information is available at the end of the article
}

women experiencing physical, sexual or psychological violence from a partner has increased gradually, approximately $19 \%$ to $33 \%$, over the ten-year period of 1998-2008 [1,2].

The law for the prevention of spousal violence and the protection of victims, enacted in Japan in 2001, called for the identification of abused women and for providing adequate resources such as support centres or hotlines. The language used in the law indicates that health care providers may report IPV to the police and support centres, taking into consideration the intention of the victim, however reporting is not mandated. Providing women with access to counselling centres is a clear intent of the law. Despite the law, most health care 
providers are not adequately trained to carry out this role [3], nor is abuse screening, or even inquiry, routine in the healthcare settings, therefore safety planning and referral to community resources are not provided. It is also likely that many health professionals do not recognize most incidents of IPV and therefore do not contribute to the woman's health recovery.

According to a recent survey in Japan, the prevalence of IPV during pregnancy was around 5\% [4]. IPV affects women and infant's health and pregnancy outcomes: gynecological symptoms [5], sexually transmitted diseases, abortion, and complications of pregnancy: diabetes, hypertension, infection, anemia [6-8] and low weight infants [9]. Pregnancy is one of the few opportunities where healthy women come into contact with their own health care providers. Therefore, IPV screening and assessment in prenatal settings should be standard care for all pregnant women. Although there is insufficient evidence to support the effectiveness of interventions after universal screening $[10,11]$, screening, carefully orchestrated early intervention attempts during pregnancy for IPV are still imperative because of the high risk of substantial damage to the health of women and children. In order to start effective interventions for abused women in Japan, the appropriate timing and method of IPV screening in healthcare settings needs to be better understood.

Even though researchers developed valid and reliable screening instruments [12], their effective implementation is still controversial. Researchers have conducted some randomised controlled trials (RCT) to compare screening methods. MacMillan et al. examined optimal screening methods (computer, written, face-to-face) for IPV in emergency departments, family practice and women's health clinics. They concluded that no statistically significant differences were found for screening methods, however, women preferred the self-administered method [13]. Another RCT in family practice also identified no statistically significant difference between interviews and self-administered questionnaires [14]. Of the two RCTs conducted in emergency departments, one demonstrated that computerized screening increased the identification rate of abused women compared to a questionnaire [15]; the other found that an audiotape questionnaire detected more abused women than a questionnaire [16].

All RCTs were conducted in North America; no studies were undertaken in Asian countries or in prenatal settings. The issue of cultural sensitivity remains unexplored. It is not known whether approaches used in North American are relevant in Asian countries. While the names of the cultural values may be similar, such as respect, trust and caring, the order and meaning of the values and the way they are enacted may differ, thus requiring different approaches in screening for domestic violence. Therefore we conducted a randomised controlled trial in a prenatal clinic in Japan to compare the rate of IPV disclosure based on face-to-face interviews and a self-administered questionnaire and to identify responses to screening experiences.

\section{Methods}

This was a parallel group, randomized controlled treatment study. The study took place from February through November 2003 at the prenatal clinic of a general hospital, in a typical urban area of Tokyo, which had approximately 1500 deliveries each year.

\section{Participants}

Eligible pregnant women were consecutively selected between February 22 and May 30, 2003. At the time of recruitment most were at 14-15 weeks of pregnancy, although a few were close to 25 weeks, which was the cut-off point for study eligibility. Research assistants, who had master's degrees in nursing, or the researcher (YK) invited eligible women to privately discuss their participation in the study. They were informed in detail (following the Declaration of Helsinki) regarding their right to confidentiality, to withdraw from the study at any time without jeopardizing their care and protection of anonymity if results were published or presented. The women were asked if they were Japanese speaking, had any serious mental diseases and if their partner would agree to leave the room during the interview or while completing the questionnaire. Children under two years old were allowed to stay with their mother, but older children, who might report the discussion about IPV to the male partner or family members [17], were not allowed to stay and instead were provided with toys and a separate place to play. All participants gave written informed consent.

\section{Randomization}

After informed consent, the participants were randomised, by means of numbered sealed envelopes, to either the interview or the questionnaire group. We used a random number table in blocks of four to ensure that approximately equal numbers of women were allocated to each group. Because of the nature of the screening methods participants could not be blinded to the group assignment. The same researcher performed the allocation procedure and data analysis.

\section{Instruments}

Two methods of implementing the screening tool, Violence Against Women Screen (VAWS), were compared in this trial: self-administered and face-to-face interview. VAWS is a 7-item Japanese screening instrument. Items 
include information about: couple's relationship, perceived behavior of the partner (i.e. difficultly settling arguments by talking it through; becoming frightened, being yelled at, partner hitting the wall or throwing objects; being forced into sex; being pushed or slapped; being hit or kicked in that order). Item responses are on a 3-point Likert scale: often (2), sometimes (1) or never (0). The scores range from 0 to 14 and a score of 2 or above indicates IPV. The sensitivity of VAWS is $86.7 \%$ and specificity is $80.2 \%$, based on the Japanese version of the index of spouse abuse [18].

Secondary outcomes were comfort level and the need to consult with the nurse after screening, which were used as indicators of possible adverse effects of IPV screening. The comfort level question referred to their sense of ease or discomfort. We asked women who had used either method: self-administered questionnaire or interview: "How did you feel being questioned about your relationship with your partner". They indicated their comfort level using a 4-point Likert rating: 1 (not at all comfortable) to 4 (very comfortable). In order to measure their desire for consultation about their relationship, we asked women: "Would you like to consult with a nurse when you have a problem with your husband?" The response options were: yes, no or cannot decide.

Prior to administrating the first screening, all participants responded to a written questionnaire covering demographic information such as age, education, employment, marital status, alcohol and smoking habits before pregnancy, and lifetime experience of physical violence from a former partner.

\section{Screening and consultation protocol}

All participants received three screenings: the first screening was upon entry to the study at 14-25 weeks of pregnancy; the second screening was at 20-30 weeks and the third screening was at 35 weeks or more. In the first screening, women reported about abuse for the "past 12 months", but at screenings two and three, they reported about abuse: "since the last time you were interviewed" [19]. Three assessments were made because disclosure about abuse could occur at any time during pregnancy especially after establishing a trusting relationship with a nurse.

The interviewers were three female nurses working at the hospital outpatient department. The researcher provided three training sessions in order to assure interviews proceeded in accordance with the study protocol and with an awareness of IPV as an issue for women. The first training session was a 2-hour lecture about the prevalence, dynamics and the physical and psychological impact of IPV, and appropriate care for abused women. A survivor of IPV who told her story of recovery conducted the second session. Theories (i.e. cycle of violence and learned helplessness) were discussed to explain why she did not escape from her partner. In the third session, the researcher trained the nurses on the use of the protocol for screening and intervention for abused women in the prenatal setting.

Interviews were conducted at the prenatal clinic in a private room that was partitioned into three cubicles or in a private area. The nurse sought to establish a partnership with the woman, based on the women centered care model [20] of respect and empowerment. Half of the women were interviewed by the same nurse at all three screenings. The nurse read the VAWS questions to the woman, while the woman also read the questionnaire.

If the woman screened positive and wanted the nurse to report the abuse to a support centre or to the police, the nurse would make the report. In addition, the nurse discussed with the woman how to maintain her safety and attempted to aid the woman in decision-making. The nurse also gave the woman a community resource card with information about a crisis hotline and local shelters. Women in the questionnaire group completed the VAWS in the official prenatal clinic interview room where the same type of resource cards were available. The resource cards were also available in the waiting area. The abuse consultants' contact information was listed in the questionnaire for easy access. Thus, although all the women had access to IPV resources the women in the interview group may have received additional assistance compared to the women in the questionnaire group.

\section{Data Collection}

The primary outcome was the disclosure rate of abuse by women who were identified by the VAWS tool during the three screening sessions compared to those in the interview group. We counted single disclosures only once, at the first or second or third screenings. We also included the pattern of multiple disclosures at screenings: first and second; first and third; second and third and first, second and third. This provided us with unduplicated accounts of abuse disclosures.

\section{Sample Size and Statistical Analysis}

From the survey, we estimated the baseline proportion of physical violence cases during pregnancy to be $3 \%$ [4], so we aimed to recruit 140 women per group in order to detect a difference between groups at a $7 \%$ effect size, based on previous research $[19,21]$ with $80 \%$ power. Extra women were recruited to account for attrition, which was expected to be $10-20 \%$.

All analyses were carried out using SPSS for Windows $11.5 \mathrm{~J}$ (Chicago, IL, USA). Categorical differences between the two groups were tested with the $\chi^{2}$ test or 
Fisher's exact test. Multiple logistic regression analysis was performed to adjust for potential baseline differences in the characteristics between the groups. For each group we calculated the IPV positive screen rate using a 95\% confidential interval (CI). All statistical tests were performed with a two-sided $5 \%$ level of significance. All analyses were done using an intention-totreat basis, which included all randomized participants. The Ethics Committee of St.Luke's College of Nursing approved the protocol.

\section{Results}

Sample and Demographic Characteristics, and Retention Rates

During the study period, 382 pregnant women made an appointment at the hospital between 14 and 25 weeks of their pregnancy (Figure 1). Of these, 355 (92.9\%) met all eligibility criteria; eight were lost to follow-up and 19 refused to participate, the remaining 328 eligible women were randomly assigned to either the interview or questionnaire group. With the exception of smoking, there were no significant differences between the groups at baseline (Table 1) and the primary outcome was adjusted to account for this. In the interview group, 148 (89.7\%) participants completed all three screenings and 17 withdrew because of: referral to university hospitals $(n=3)$; moving out of the area $(n=9)$; preterm delivery $(n=1)$ or refusal to continue the screening interview $(n=2)$. In the questionnaire group, 149 (91.4\%) participants completed all three screenings and 14 withdrew because of moving out of the area $(n=13)$ or preterm delivery $(n=1)$. Retention rates did not differ between the two groups.

\section{Primary Outcome}

For the interview group the identification rate was $32 / 165$ (19.4\%) compared with $48 / 163$ (29.4\%) in the questionnaire group, which was much higher (relative risk $0.66,95 \%$ CI 0.46 to 0.97 ), even when controlled for smoking (adjusted odds ratio 0.59 , 95\% CI 0.35 to 0.98 ).

The numbers of abused women disclosing abuse at each screening are shown in Table 2 . Of the 32 women in the interview group, 16 (50.0\%) disclosed abuse only at the first screening compared to seven (14.6\%) from the questionnaire group. There was no discernable disclosure pattern between the two groups or within each group.

\section{Secondary Outcomes}

The comfort level during screening for DV did not differ between the two groups: 98 (68.1\%) women in the interview group and $88(60.7 \%)$ in the questionnaire group responded that they were "very comfortable" with the screening for DV. The rate of those needing consultation with a nurse after the screening was similar and relatively low in both groups (interview $11.1 \%$; questionnaire $11.7 \%)$.

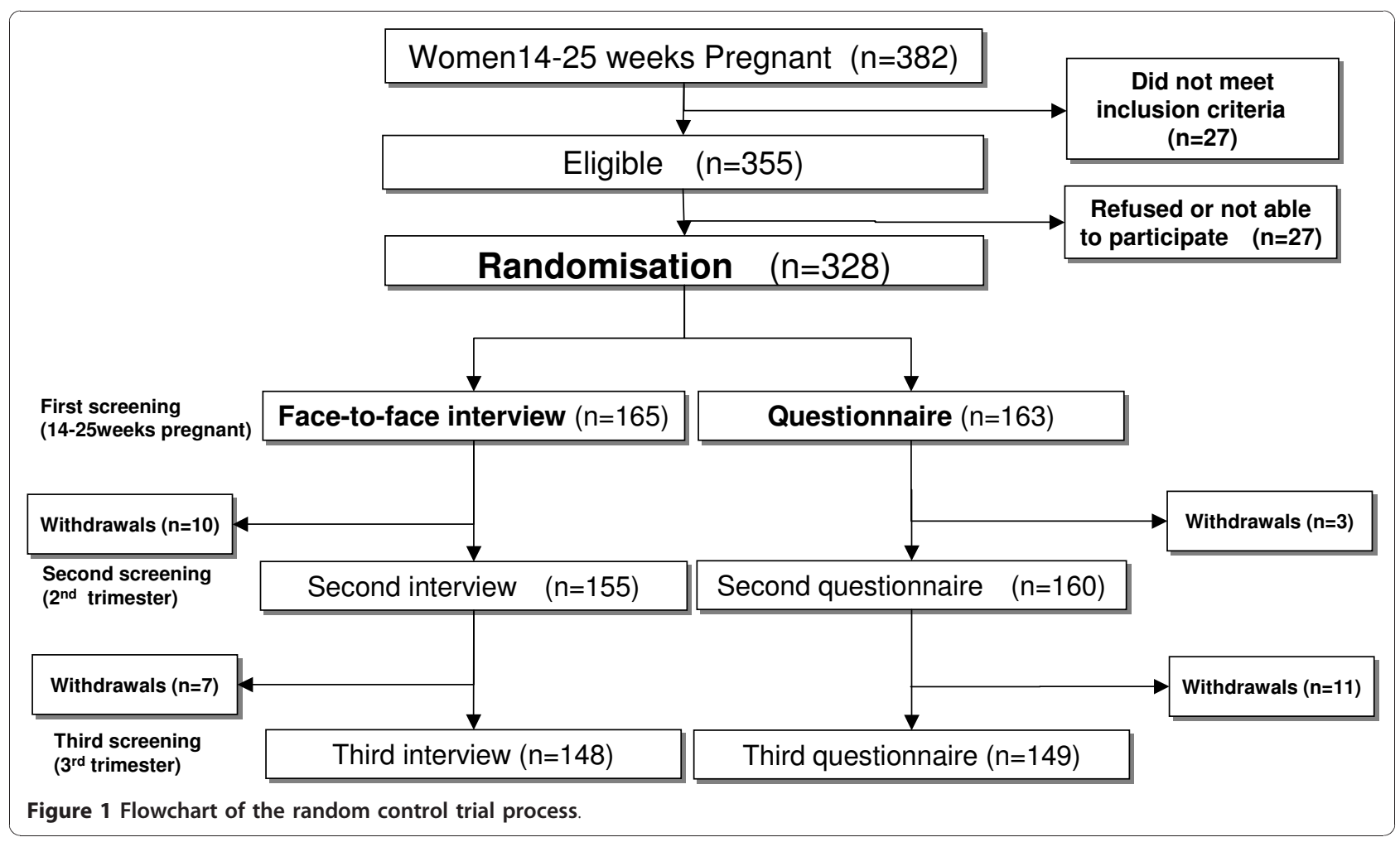


Table 1 Demographic characteristics of the women at first screening during the first trimester of pregnancy ( $N$ = 328)

\begin{tabular}{|c|c|c|}
\hline & $\begin{array}{c}\text { Face to face } \\
\text { interview group } \\
(n=165)\end{array}$ & $\begin{array}{c}\text { Questionnaire } \\
\text { group } \\
(n=163)\end{array}$ \\
\hline \multicolumn{3}{|l|}{ Age (years) } \\
\hline 20-29 & $47(29.0)$ & $53(32.7)$ \\
\hline $30-39$ & $111(68.5)$ & $106(65.4)$ \\
\hline$\geq 40$ & $7(2.5)$ & $3(1.9)$ \\
\hline \multicolumn{3}{|l|}{ Parity } \\
\hline Primipara & $102(62.6)$ & $105(64.4)$ \\
\hline Multipara & $61(37.4)$ & $58(35.6)$ \\
\hline \multicolumn{3}{|l|}{ Education } \\
\hline $\begin{array}{l}\text { Junior high/High school } \\
\text { graduate }\end{array}$ & $20(12.3)$ & $24(14.8)$ \\
\hline Junior college graduate & $74(45.7)$ & $69(42.6)$ \\
\hline $\begin{array}{l}\text { College graduate/ } \\
\text { Postgraduate }\end{array}$ & $68(42.0)$ & $69(42.6)$ \\
\hline \multicolumn{3}{|l|}{ Marital status } \\
\hline Married & $159(98.1)$ & $157(97.5)$ \\
\hline Single & $3(1.9)$ & $4(2.5)$ \\
\hline \multicolumn{3}{|l|}{ Employment } \\
\hline Full time & $53(32.7)$ & $58(36.40)$ \\
\hline Part time & $32(19.8)$ & $26(16.1)$ \\
\hline Not working & $77(47.5)$ & $77(46.9)$ \\
\hline \multicolumn{3}{|l|}{ Alcohol before pregnancy } \\
\hline Yes & $109(67.3)$ & $115(71.0)$ \\
\hline No & $53(32.7)$ & $47(29.0)$ \\
\hline \multicolumn{3}{|l|}{ Smoking before pregnancy } \\
\hline Yes & $42(25.9)$ & $27(16.7)$ \\
\hline No & $120(74.1)$ & $135(83.3)$ \\
\hline \multicolumn{3}{|l|}{$\begin{array}{l}\text { Lifetime experience of physical } \\
\text { violence by male partner }\end{array}$} \\
\hline Yes & $8(4.8)$ & $11(6.8)$ \\
\hline No & 154 (95.1) & $151(93.2)$ \\
\hline
\end{tabular}

Values are number (percentage).

\section{Discussion}

Implications of the Findings

The results of a RCT conducted to determine which of two screening methods (face-to-face interview or selfadministered questionnaire) was more effective, in detecting woman who experienced abuse from their partner, in the prenatal setting in Japan indicated that the questionnaire method identified more abused women than the interview.

Previous studies of obstetric patients demonstrated higher disclosure with written questionnaires compared with face-to-face interviews [21,22], although other RCTs reported no difference among methods $[13,14]$. The results of our study concur with those of Caterino et al. [21] and Webster et al. [22] who studied pregnant women as did this study. Pregnant women usually come to the prenatal clinics with their partners, so they are more cautious about privacy. This is the main reason that a written questionnaire is optimal for pregnant women especially in Japan, a very homogeneous country where the literacy rate is almost $100 \%$. In addition the VAWS is written in an easy form of Japanese.

Cultural reasons must also be considered as to why the self-administered questionnaire was the better method for pregnant women to identify IPV in this prenatal setting. First, for Japanese, maintaining harmony in a group is a strong value; therefore open communication is discouraged because of the group orientation [23]. There is also a strong value of keeping family matters private and inner feelings hidden [23]. This means it is difficult to disclose private matters verbally to another person, especially concerning problems within a family, even if the other person is a health professional. With regards to IPV, women tend to feel shame and guilt, so disclosing though a face-to-face interview is even more difficult. In contrast, privately filling out the written

Table 2 Number of women disclosing abuse at each screening by group

\begin{tabular}{|c|c|c|c|c|c|c|c|}
\hline Time of disclosure & $\begin{array}{l}\text { Face to face } \\
\qquad(\mathrm{n}=\end{array}$ & $\begin{array}{l}\text { interview group } \\
=165 \text { ) }\end{array}$ & $\begin{array}{l}\text { Question } \\
\text { (n }\end{array}$ & $\begin{array}{l}\text { nare group } \\
=163 \text { ) }\end{array}$ & Relative risk & $(95 \% \mathrm{Cl})$ & $P$ value* \\
\hline Total no. abused women in the 3 screenings & 32 & & 48 & & 0.66 & $(0.45-0.97)$ & 0.034 \\
\hline First screening only & 16 & $(50.0)$ & 7 & $(14.6)$ & & & \\
\hline Second screening only & 1 & $(3.1)$ & 5 & $(10.4)$ & & & \\
\hline Third screening only & 3 & $(9.4)$ & 1 & $(2.1)$ & & & \\
\hline First and second screening & 2 & $(6.3)$ & 10 & $(20.8)$ & & & \\
\hline First and third screening & 3 & $(9.4)$ & 4 & (8.3) & & & \\
\hline Second and third screening & 1 & (3.1) & 4 & (8.3) & & & \\
\hline First, second and third screening & 6 & $(18.8)$ & 17 & $(35.4)$ & & & \\
\hline
\end{tabular}

Values are number (percentage) unless stated otherwise

Numbers are unduplicated

${ }^{*} \chi^{2}$ test 
questionnaire might seem easier and more secure when revealing partner violence. Second, healthcare settings in Japan still lack privacy; for example, waiting rooms are crowded and there are few rooms for private consultation. Even in this study, in which the privacy of the women during the interview was our first concern; we provided privacy but could not always provide a private room. Finally, the face-to-face interview requires ongoing training and extra time; it would require a national level initiative to establish that resource within prenatal settings.

We found that the proportion of new, ended and ongoing cases was different between the two groups. About one third of abused women in the questionnaire group reported violence over time. By contrast half of abused women in the interview group reported violence only at the first screening. Previous studies revealed it was difficult to change the situation or relationship between a couple, and to stop the violence in this short period of time [24]; therefore it is possible that some women were reluctant to continue to admit to being abused. Social desirability effect might be the cause of this difference. In addition although most of the women who were interviewed said that they felt "comfortable" with the screening, they might have responded differently if there were more questions about aspects of comfort. Finally, previous studies revealed that the effect of training was not maintained 12 months later [25]. The effect of training was not evaluated in the present study and the care for the women may have changed over the 10-month study period so that the interviewers were less supportive during the interview.

It is important to ensure that pregnant women feel safe answering IPV screening questions. In Japan, we recommend that women screened as positive discuss their situation and feelings after the self-administered questionnaire screening. Identifying abused women enables the IVP trained healthcare provider to intervene, providing both the woman and fetus with a safer environment. The nursing care protocol for abused women that was used in this study needs to be further developed in order to improve prenatal care in Japan.

\section{Study Limitations}

First, the study protocol excluded women who: were nonJapanese speakers; had a serious mental illness; did not agree to leave their partner during the interview, and an additional 27 women who refused to participate. This may have generated a self-selection bias eliminating women who were at high-risk for DV leading to underestimation of its prevalence. Second, measurements of primary and secondary outcomes had psychometric property limitations. The VAWS was the first Japanese IPV screening instrument that was developed with respect to culture and social situation. Although the sensitivity of the VAWS was comparatively high, specificity was low compared with other tools such as the: 'Hurts, Insults, Threatens and Screams at Her' (HITS), Women's Experience with Battering Scale (WAST) and Partner Violence Screen (PVS). We need additional valid and reliable Japanese screening tools to test the screening methods. Measurements of secondary outcomes were also limited by their reliability and validity. The concepts of 'comfort' and 'need' require further development and testing within the context of the Japanese prenatal setting. A single question might be not enough to measure the comfort level and needs, even though Sauro \& Dumas found acceptable reliability and validity with one-question Likert tests [26]. We will have to detect if adverse effects occurred in one method of screening more than the other. Third, this study was conducted at an urban prenatal clinic. Further studies are needed in different geographical areas and in different types of healthcare settings, such as emergency departments or mental health care clinics, to establish the generalization of the results. However, these limitations do not detract from the important finding of this study, which is that a self-administered questionnaire resulted in more disclosure of IPV in a Japanese prenatal setting. Finally, although the study was conducted in 2003, the data remains useful and fortunately still quite timely. These findings should encourage researchers in Asian countries to replicate and extend the research.

\section{Conclusion}

In Japan, within a perinatal setting, the self-administered questionnaire compared to the face-to-face interview detected significantly more women who experienced abuse from their partners. The comfort level and the need for consulting with a nurse after the screening did not differ between the two groups and must be interpreted with caution because of the beginning stage of tool development. In conclusion the self-administered questionnaire was an appropriate method for identifying abuse in a Japanese prenatal setting when conducted with privacy safeguards and resource follow-up.

\section{Acknowledgements}

This study was supported by Grant-in-Aid for COE Research, provided by the Ministry of Education, Culture, Sports, Science and Technology of Japan. The authors thank Caroline White RN PhD and Sarah Porter RN PhD who provided English editing services.

\section{Author details}

${ }^{1}$ St Luke's College of Nursing, 10-1 Akashi-cho, Chuo-ku, Tokyo 104-0044, Japan Associate Professor in nursing and midwifery. ${ }^{2}$ Department of Medicine, Pharmacoepidemiology, University of Tokyo, 7-3-1 Hongo, Bunkyoku, Tokyo 113-0033, Japan. 


\section{Authors' contributions}

YK carried out the data collection and drafted the manuscript. HE participated in the sequence alignment. YY participated in the design of the study and performed the statistical analysis. SH conceived of the study, and participated in its design and coordination and helped to draft the manuscript. All authors read and approved the final manuscript.

\section{Competing interests}

The authors declare that they have no competing interests.

Received: 7 April 2010 Accepted: 24 December 2010

Published: 24 December 2010

\section{References}

1. Gender Equality Bureau, Cabinet Office: The survey of spouse violence. 2008 [http://www.gender.go.jp/e-vaw/chousa/h2103top.html].

2. Gender Equality Bureau, Cabinet Office: The survey of spouse violence. 1999 [http://www.gender.go.jp/e-vaw/chousa/09.html].

3. Kataoka Y, Shitaya E, Kano N, Otake M: Nursing care of female victims of sexual assault and violence: a hospital-based questionnaire survey. J St Luke's Soc Nurs Res 2005, 9:1-10.

4. Kataoka Y, Yaju Y, Eto H, Horiuchi S: Domestic violence against women during pregnancy. Nippon Koshu Eisei Zasshi 2005, 52(9):785-795.

5. Greenberg EM, McFarlane J, Watson MG: Vaginal bleeding and abuse: assessing pregnant women in the emergency department. MCN Am J Matern Child Nurs 1997, 22(4):182-186.

6. Amaro H, Fried LE, Cabral H, Zuckerman B: Violence during pregnancy and substance use. Am J Public Health 1990, 80(5):575-579.

7. Berenson AB, Wiemann CM, Wilkinson GS, Jones WA, Anderson GD: Perinatal morbidity associated with violence experienced by pregnant women. Am J Obstet Gynecol 1994, 170(6):1760-1766, discussion 1766-1769.

8. McFarlane J, Parker B, Soeken K: Abuse during pregnancy: associations with maternal health and infant birthweight. Nurs Res 1996, 45(1):37-42.

9. Murphy CC, Schei B, Myhr TL, Du Mont J: Abuse: a risk factor for low birth weight? A systematic review and meta-analysis. CMAJ 2001, 164(11):1567-1572.

10. MacMillan $\mathrm{HL}$, Wathen $\mathrm{CN}$, Jamieson E, Boyle MH, Shannon HS, FordGilboe M, Worster A, Lent B, Coben JH, Campbell JC, McNutt LA: Screening for intimate partner violence in health care settings: a randomized trial. JAMA 2009, 302(5):493-501.

11. Ramsay J, Carter Y, Davidson L, Dunne D, Eldridge S, Feder G, Hegarty K, Rivas C, Taft A, Warburton A: Advocacy interventions to reduce or eliminate violence and promote the physical and psychological wellbeing of women who experience intimate partner abuse. Cochrane Database Syst Rev 2009, 8(3):CD005043.

12. Feder G, Ramsay J, Dunne D, Rose M, Arsene C, Norman R, Kuntze S, Spencer A, Bacchus L, Hague G, Warburton A, Taket A: How far does screening women for domestic (partner) violence in different healthcare settings meet criteria for a screening programme? Systematic reviews of nine UK National Screening Committee criteria. Health Technol Assess 2009, 13(16):iii-iv, xi-xiii, 1-113, 137-347.

13. MacMillan HL, Wathen CN, Jamieson E, Boyle M, McNutt LA, Worster A, Lent B, Webb M, McMaster Violence Against Women Research Group: Approaches to screening for intimate partner violence in health care settings: a randomized trial. JAMA 2006, 296(5):530-536.

14. Chen PH, Rovi S, Washington J, Jacobs A, Vega M, Pan KY, Johnson MS: Randomized comparison of 3 methods to screen for domestic violence in family practice. Ann Fam Med 2007, 5(5):430-435.

15. Rhodes KV, Drum M, Anliker E, Frankel RM, Howes DS, Levinson W: Lowering the threshold for discussions of domestic violence: randomized controlled trial of computer screening. Arch Intern Med 2006, 166(10):1107-1114.

16. Bair-Merritt MH, Feudtner C, Mollen CJ, Winters S, Blackstone M, Fein JA: Screening for intimate partner violence using an audiotape questionnaire: a randomized clinical trial in a pediatric emergency department. Arch Pediatr Adolesc Med 2006, 160(3):311-316.

17. Zink T: Should children be in the room when the mother is screened for partner violence? J FamPract 2000, 49:130-136.

18. Kataoka Y: Development of the Violence Against Women Screen. J Jpn Acad Nurs Sci 2005, 25:51-60
19. McFarlane J, Bateman L, Bullock L, Christoffel K, Miller V: Assessing for abuse: self-report versus nurse interview. Public Health Nurs 1991, 8:245-250.

20. Horiuchi S, Kataoka Y, Eto H, Oguro M, Mori T: The applicability of womencentered care: two case studies of capacity-building for maternal health through international collaboration. Japan Journal of Nursing Science 2006, 3:143-150

21. Canterino JC, Ananth CV, Harrigan JT, VanHorn LG, Vintzileos AM: Domestic abuse in pregnancy: a comparison of a self-completed domestic abuse questionnaire with a directed interview. Am J Obstet Gynecol 1999, 181:1049-1051.

22. Webster J, Holt V: Screening for partner violence: direct questioning or self-report? Obstet Gynecol 2004, 103:299-303.

23. Doi T: The anatomy of dependence. Tokyo: Kodansha International Ltd 1971, (J. Bester, trans.).

24. Smedslund G, Dalsbø TK, Steiro AK, Winsvold A, Clench-Aas J: Cognitive behavioural therapy for men who physically abuse their female partner. Cochrane Database Syst Rev 2007, 3: CD006048.

25. Fanslow JL, Norton RN, Robinson EM: One year follow-up of an emergency department protocol for abused women. Aust N Z J Public Health 1999, 23:418-420, 24

26. Sauro J, Dumas LS: Comparison of three one-question, post-task usability questionnaires. 2009 [http://www.measuringusability.com/papers/ Sauro_Dumas_CHI2009.pdf], Retrieved July 29 from.

\section{Pre-publication history}

The pre-publication history for this paper can be accessed here: http://www.biomedcentral.com/1471-2393/10/84/prepub

doi:10.1186/1471-2393-10-84

Cite this article as: Kataoka et al:: Self-administered questionnaire versus interview as a screening method for intimate partner violence in the prenatal setting in Japan: A randomised controlled trial. BMC Pregnancy and Childbirth 2010 10:84.

\section{Submit your next manuscript to BioMed Central and take full advantage of:}

- Convenient online submission

- Thorough peer review

- No space constraints or color figure charges

- Immediate publication on acceptance

- Inclusion in PubMed, CAS, Scopus and Google Scholar

- Research which is freely available for redistribution

Submit your manuscript at www.biomedcentral.com/submit
C) Biomed Central 